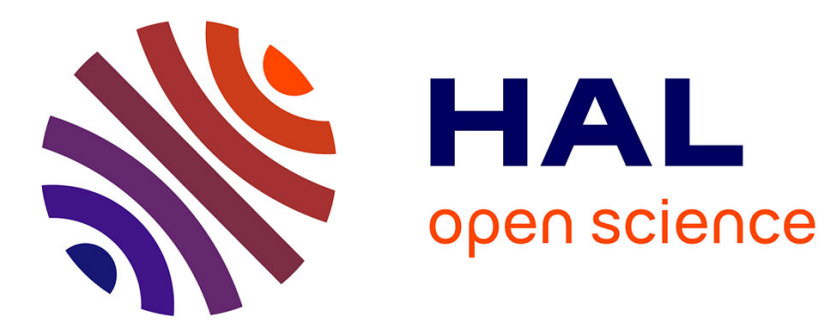

\title{
Sur le diamagnétisme des ions
}

Pierre Weiss

\section{To cite this version:}

Pierre Weiss. Sur le diamagnétisme des ions. J. Phys. Radium, 1930, 1 (6), pp.185-193. 10.1051/jphysrad:0193000106018500 . jpa-00205421

\section{HAL Id: jpa-00205421 https://hal.science/jpa-00205421}

Submitted on 1 Jan 1930

HAL is a multi-disciplinary open access archive for the deposit and dissemination of scientific research documents, whether they are published or not. The documents may come from teaching and research institutions in France or abroad, or from public or private research centers.
L'archive ouverte pluridisciplinaire HAL, est destinée au dépôt et à la diffusion de documents scientifiques de niveau recherche, publiés ou non, émanant des établissements d'enseignement et de recherche français ou étrangers, des laboratoires publics ou privés. 


\title{
LE JOURNAL DE PHYSIQUE
}

$\mathbf{B} \mathbf{T}$

\author{
LE RADIUM
}

\section{SUR LE DIAMAGNÉTISME DES IONS}

\author{
par M. Pierre WeisS.
}

\begin{abstract}
Som naire. - Puur soum tttre les théories du diamagnétisme au contrôl ; de l'expérience li importe de se procurer des valeurs exactes du diamagnétisme des ions.

On peut déduire les coefficients d'aimantation des ions des mesures sur les sels en partant de la valeur nulle du diamagnétisme de l'ion $\mathrm{H}+$. à condition de tenir compte de l'altération de l'eau dans laquelle il est dissous et qui lui confère un paramagnétisme apparent. Cette altération et d'une manière plus générale les petites déformations qu'ions el molécules neutres subissent de la part des ions qui les approchent, en solution et dans le cristal, peuvent être évaluées au moyen des donnés réfractométriques correspondantes. On s'appuie sur le fait, donné par l'expérience et conforme aux théories, que le diama. gnétisme est moitié moins sensible à une petite déformation que la réfraction.

On déduit des mesures très précises de Hocart les coefficients d'aimantation de quelques ions à l'état libre. Ces déterminations sont appuyées par les valeurs de Re'cheneler et de Pascal. L'accord entre l'expérience et les théories laisse à désirer.
\end{abstract}

Théories et possibilité de contrôle. - On possède deux théories récentes du diamagnétisme qui comme celle de Langevin le déduisent de la précession de Larmor. L'une et l'autre reposent sur la répartition des charges électriques dans l'espace autour du centre positif donnée par la théorie de Schroedinger.

Pauling ( ${ }^{1}$ ) traite le cas de l'atome du type hydrogène, possédant diverses valeurs de la charge centrale $Z$ et où l'orbite de l'électron unique est caractérisée par les nombres quantiques. Il en déduit le diamagnétisme des atomes plus compliqués en corrigeant la charge $Z$ au moyen d'un effet écran variable avec l'étage etavec $Z$ et dansl'évaluation duquel il fait intervenir les propriétés de la réfraction.

Stoner $\left(^{2}\right)$ évite los approximations faites par Pauling dans la réduction à l'atome du type hydrogène. Il s'appuie sur la répartition des charges calculée directement par Hartree $\left(^{3}\right)$ au moyen de la théorie de Schroedinger pour des atomes plus compliqués.

Le diamagnétisme des molécules est beaucoup moins abordable. M. G. Foëx $\left(^{4}\right)$ a insisté sur la difficulté du problème quand le mouvement des électrons est réglé par plusieurs centres positifs.

On possède déjà par les mesures de Hector et Wills le diamagnétisme de $\mathrm{He}, \mathrm{Ne}, \mathrm{A}\left(^{(3}\right)$.

(1) Proc. Roy. Soc., t. 114 (1927), p. 181, voir aussi vax Vlegk. Phys. Rev., vol. 31 (1928), p. 587.

(2) Proc. Leeds Phil. Soc., vol. 1 (1929), p. 484.

(3) Proc. Camb. Phil. Soc., t. 24 (1928), pp 89 et 111.

(4) J. Phys., t. $10(19: 9)$, p. 421.

(5) Phys. Rev., vol. 24 (192'), p. 418.

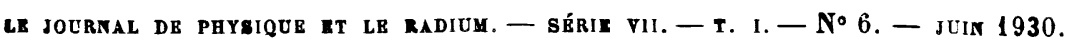


Il importe donc, pour un contròle plus étendu des théories, de demander à l'expérience des valeurs précises du diamagnétisme des ions.

Les coefficients d'aimantation données pour les sels sont en général très divergents ( $\left.{ }^{1}\right)$. Je me servirai ici, pour déterminer le diamagnétisme des ions, des mesures de Hocart $\left(^{2}\right)$ peu nombreuses mais faites par des méthodes magnétiques éprouvées et en portant une grande attention au problème chimique. Elles ont été assez précises pour mettre en évidence la différence entre le diamagnétisme des sels dissous et cristallisés. Leur accord avec les mesures de Pascal $\left(^{3}\right)$ dans les limites de la précision recherchée par les deux auteurs suffit à écarter la supposition d'erreurs de quelque importance.

Hypothèse de Wasastjerna et de Reicheneder. - La mesure du diamagnélisme des sels donne la somme des diamagnétismes des deux ions. Il faut se procurer par ailleurs le diamagnétisme d'un premier ion pour calculer tous les autres par différence. Le mème problème s'est posé dans la détermination de la réfraction des ions à partir de celle des sels et W'asastjerna $\left({ }^{4}\right)$ a proposé de la résoudre en admettant que l'ion II + , formé d'un proton 5 ans électron planétaire a une réfractian nulle. Mais Eajans et Joos (") ont montré que l'on commettrait ainsi une erreur grave à cause de l'altération que l'ion $\mathrm{H}+$ fait subir aux molécules du solvant. En fait, dans leur étude détaillếe des réfractions des ions, ils ont procédé par une répartition hypothétique de la réfraction de NaF entre l'anion et le cation. Joos $\left(^{3}\right)$ tente aussi une répartition du diamagnétisme de $\mathrm{KCl}$ entre $\mathrm{K}+$ et $\mathrm{Cl}-$ et donne le premier un tableau de diamagnétismes ioniques.

Mais tandis que Eajaus et Jaos aboatissent à un ensemble cohérent de réfractions, l'insulfisance des domnées numériques empẽche Joos de réussir ell diamagnétisme, Mème actuellement les données sont trop incomplètes pour qu'on puisse suivre la voie qu'il a indiquée.

Reicheneder ${ }^{(i)}$ a fait lihypothèse de la nullité du diamagnétisme de $\mathrm{H}^{-}$. Mais l'analogie avec la réfraction apprend qu'elle est insuffisante si elle n'est complétée par l'évaluation de l'altération du diamagnétisme de l'eau par l'ion $\mathrm{H}^{+}$.

La réfraction comme moyen d'étude de fa déformation des ions. - On peut obtenir les renseignements qui manquent en diamagnétisme par une transposition des propriétés de la réfraction. Les deux phénomènes dépendent en effet en première ligne des orbites extérieures et sont influencés par les déformations que les champs électriques émanant des ions font subir aux orbites d'autres ions ou de molécules qu'ils approchent.

Fajans et Joos (') ont montré que lorsqu'une charge positive approche d'un ion ou d'une molécule neutre elle opère un resserrement des orbites et par conséquent une diminution de la réfraction. L'approche d'un ion négatif produit. un relàchement des orbites et une augmentation de la réfraction.

Liion positif (par exemple $\mathbf{K}^{+}$) étant plus petit que l’ion négatif (par exemple $\mathrm{Cl}^{-}$) ayant

(1) Voici, par exemple, quelques valeurs récentes du foefficient d'aimantation du chIorure de potassium :

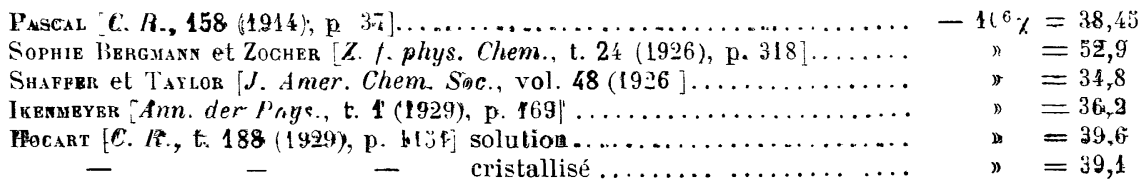

(1) $C$ R., t. $188(19$ I9), p lint.

(2) C R., t. 158 (1914), p. 3 i.

(3) $Z$ fir phy.s. Chems, t. 101 (1922), p 193.

(4) Z. fur Phys, t. 23 (1924), p. 1 .

(i) Z. fuir Phys.. t. 19 (1923), p 3 iz et t. $32(1929) \times$ p. \&.

( $\left.{ }^{6}\right)$ Ann. der Phys., 5้อ s, t. $3(1929)$, p. 58.

(i) Loc. cit. Yoir aussi Fajans, Konnex et Giffiex. $Z$ f. Elektrochemie (1928) \&. 1. 
les mèmes éteatrons et ne différant de lui que par la charge centrale, altère lavantage les molécules ou ions voisins. L’ion négatif dont les orbites sont plus làches est plus altérable que l'ion positif. Le rapprochement étroit des ions contraires lans le cristal produit des déformations plus fortes que le contact plus làche des ions avec les molécules neutres du solvant. La réfraction et le diamagnétisme sont influencés par ces déformations, variables de l'état liquide à l'état existallisé et, dans le cristal. avec la nature de l'autre ion. Ne sont strictement additifs que les réfractions et les diamagnétismes en solution étendue, altération de l'eau comprise.

Puiaque, comme nous le verrons, le diamagnétisme est moins sensible aux déformations que la réfraction, on peut admettre que lorsque l'effet est négligeable pour la réfraction, il l'est aussi pour le diamagnétisme. D'après Fajans et Joos les ions $\mathrm{H}^{+}, \mathrm{Li}^{+}, \mathrm{Na}^{+}$, $\mathrm{Mg}^{++}, \mathrm{Sr}^{++}, \mathrm{Al}^{++}+, \mathrm{La}^{+++}$dissous riminuent d'une manière sensible la réfraction de l'eau, tandis que l'effet des ions plus gros, $\mathrm{K}^{+}, \mathrm{Rb}^{+}, \mathrm{Cs}^{+}, \mathrm{Ba}^{+}+$peut ètre négligé. Les ions halogènes, plus gros à masse égale, sont moins actifs et, en vertư de leur charge négative, produisent un accroissement de la réfraction de l'eau. L'effet est sensible pour $F-$ seulement et négligeable pour $\mathrm{Cl}^{-}, \mathrm{Br}-, \mathrm{I}-$. Voici les valeurs numériques des altérations de la réfraction de l'eau données par ces auteurs :

\section{TaBl,BAU I.}

$$
\begin{array}{cc}
\mathrm{H}+\ldots & -0,62 \\
\mathrm{Li}+\ldots & -0,53 \\
\mathrm{Na}+\ldots & -0,3 \\
\mathrm{~K}+\ldots & 0
\end{array}
$$

$$
\begin{array}{ll}
\mathrm{Mg}^{+}+\ldots & -1,85 \\
\mathrm{Ca}^{+}+\ldots & -0,65 \\
\mathrm{Sr}^{+}+\ldots & -0, \ddot{\mathrm{b}} \\
\mathrm{Ba}^{++}+\ldots & 0
\end{array}
$$$$
\mathrm{Al}+++. \quad-2, \ddot{8} 8
$$$$
\begin{array}{cc}
\mathrm{La}+++. & -1,9 \\
\mathrm{~F}-\ldots & +0,3 \\
\mathrm{Cl}-\ldots & 0
\end{array}
$$

Comparaison de l'altération de la réfraction et du diamagnétisme par une méme déformation. - Nous allons d'abord nous rendre compte que, qualitativement, les résultats d'Hocart sur le diamagnétisme des sels dissous et cristallisés que nous réproduisons ici sont d'accord avec ce que la réfraction fait prévoir. On a :

\section{Tableau II.}

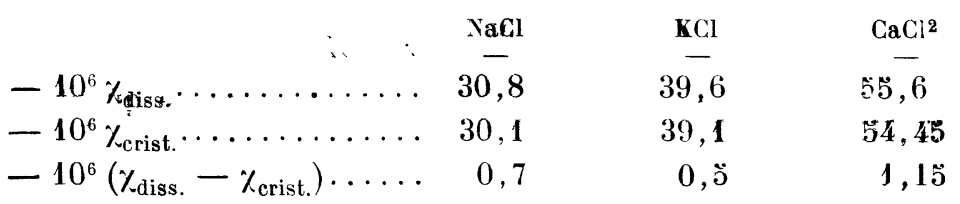

Le diamagnétisme est en effet plus grand en solution que dans le corps cristallisé. Cela exprime le fait que la perte de diamagnétisme que l'ion (léformant $\mathrm{Na}^{+}$impose à l'ron déforme $\mathrm{Cl}-$ dans le cristal est plus grande que la perte du diamagnétisme de leau par $\mathrm{Na}^{-}$dissous. Ces effets sont plus forts pour le petit ion $\mathrm{Na}^{\top}$ que pour l'ion $\mathrm{K}^{-}$, plus grand. Ils sont plas forts pour $\mathrm{Ca}^{++}$doublement chargé que pour l'ion de grandeur voisine $\mathbf{K}^{\top}$, portant une charge simple.

Nous possédons dans le cas de $\mathrm{NaCl}$ et de KGl les données numériques nécessaires pour la comparaison de la sensibilité des deux phénomènes, c'est-à-dire de l'altératiơn rełative de la réfraction et du diamagnétisme pour une même déformation. Constatons d'abord que la réfraction enseigne que dans $\mathrm{NaCl}$ et $\mathrm{KG}$ cristallisés intervient seulement l'altération de $\mathrm{Cl}^{-}$par $\mathrm{Na}^{+}$ou $\mathbf{K}^{+}$, l'altération du cation par $\mathrm{Cl}-$ étant nëgligeable ou du moins beaucoup plus faible.

Dans le cas de $\mathbf{K C l}$, où l'altération de l'eau par $\mathbf{K}^{+}$et par $\mathrm{Cl}-$ dissous esl négligeable, 
le phénomène est particulièrement simple. Il se réduit à la déformation de $\mathrm{Cl}-$ par $\mathrm{K}^{+}$dans le rapprochement des ions libres pour former le réseau cristallin.

Dans le cas de $\mathrm{NaCl}$ la différence observée se rapporte à un phénomène plus compliqué. D'une part disparaît l'altération de l'eau par $\mathrm{Na}^{+}$dissous et de l'autre apparaît la déformamation de $\mathrm{Cl}-$ par $\mathrm{Na}^{+}$dans le cristal. Mais on peut faire la correction du premier de ces effets en empruntant la donnée relative à la réfraction au tableau I et en demandant l'altération correspondante du diamagnétisme au mode de calcul que nous donnons plus loin (équation (3)). Cette anticipation est hégitime pour une correction dans la recherche d'une méthode de correction. Les éléments de la comparaison sont réunis dans le tableau III.

Tablead III.

\begin{tabular}{|c|c|c|c|c|c|c|c|c|c|c|c|}
\hline & $\begin{array}{c}R \\
\text { dissous. }\end{array}$ & $\begin{array}{c}R \\
\text { ions } \\
\text { libres. }\end{array}$ & $\begin{array}{c}R \\
\text { cristal. }\end{array}$ & $\boldsymbol{R}_{\mathrm{C} \mathrm{C}^{-}}$ & $\frac{\Delta R}{R_{\mathrm{Cl}^{-}}}$ & $\begin{array}{l}-10^{\prime \prime} \chi \\
\text { dissous. }\end{array}$ & $\begin{array}{l}-10^{5} \times \\
\text { ions } \\
\text { libres. }\end{array}$ & $\begin{array}{c}-10^{6} \chi \\
\text { cristal. }\end{array}$ & $\frac{-10^{6}}{\chi_{\mathrm{Cl}}-}$ & $\frac{-\Delta \chi}{\chi_{\mathrm{Cl}^{-}}}$ & $\begin{array}{l}\frac{\Delta R}{R_{\mathrm{Cl}^{-}}} \\
: \frac{\Delta \chi}{\psi_{\mathrm{cl}-}-}\end{array}$ \\
\hline $\mathrm{NaCl}$ & 9,2 & 9,5 & 8, ร2 & 9,07 & 0,108 & 30,8 & 31,22 & 30,1 & 2,31 & $0,0 \unlhd 3$ & 2,04 \\
\hline \multirow[t]{2}{*}{$\mathrm{KCl}$} & \multirow[t]{2}{*}{11,23} & \multirow[t]{2}{*}{11,23} & \multirow[t]{2}{*}{$10,8 \check{ }$} & \multirow[t]{2}{*}{ ) } & \multirow[t]{2}{*}{0,042} & \multirow[t]{2}{*}{39,6} & \multirow[t]{2}{*}{39,6} & \multirow{2}{*}{$\begin{array}{l}39,1 \\
\text { Moven }\end{array}$} & \multirow[t]{2}{*}{ » } & \multirow[t]{2}{*}{0,022} & 1,91 \\
\hline & & & & & & & & & & & 1,97 \\
\hline
\end{tabular}

Les variations relatives à comparer figurent dans la sixième et l'avant-dernière colonne et leur rapport dans la dernière. Les deux déterminations s'accordent mieux que la précision des mesures ne permettait de l'attendre et donnent pour la réfraction une sensibilité double de celle du diamagnétisme.

On peut aussi demander la comparaison de la sensibilité des deux phénomènes à la théorie. Elle a été faite ( ${ }^{1}$ ) pour un atome du type hydrogène, avec un électron planétaire unique dont la charge du noyau prend toutes les valeurs $Z$. On a pour cet atome :

$$
R=\frac{A}{Z^{4}} \quad \text { et } \quad \text { \% }=\frac{B}{Z^{2}}
$$

où $\mathrm{A}$ et $\mathrm{B}$ dépendent des données électroniques et des nombres de quanta caractérisant l'orbite. On en déduit :

$$
\frac{\Delta R}{R}=2 \frac{\Delta \chi}{\chi}
$$

d'accord avec le rapport des sensibilités déduit des expériences d'Hocart.

Sans doute le phénomène sur lequel porte la théorie est différent de celui sur lequel la comparaison des données expérimentales a été faite et de la déformation des molécules d'eau par les ions auquel nous allons appliquer le résultat obtenu. Mais ils ont une parenté évidente : il s'agit dans les trois cas de l'altération des orbites par le champ non uniforme d'une charge positive donnée, agissant dans des conditions telles qu'il n'y ait pas à faire intervenir un effet écran.

Paramagnétisme apparent de $\mathbf{H}^{+}$en solution. - Lorsqu'un ion dissous diminue la réfraction de l'eau d'une quantité $\lrcorner R$, la réfraction d'une molécule d'eau étant 3,76 , il détruit la réfraction de $\Delta R / 3,76$ molécules d'eau. Le diamagnétisme étant moitié moins

(') Paclivg. Proc. Roy. Soc., vol. 114 (1927), p. 181. 
Sensible, c'est celui de $\Delta R / \bar{\imath}, 52$ molécules d'eau qui disparait, et puisque $-10^{5} \%_{\mathrm{H}^{2}}=13,0$ on a :

$$
-10^{6} \Delta \%=1,73 \Delta R
$$

Les valeurs de $\lrcorner R$ sont données dans le tableau 1. Dans le cas de $\mathrm{H}^{\perp}, \Delta R=-0,62$, on a donc pour la perte de diamagnétisme, c'est-à-dire pour le paramagnétisme apparent de $\mathrm{H}+$

$$
10^{6} \%_{\mathrm{H}}+\text { diss. }=1,1
$$

Diamagnétisme de quelques ions en solution. - Hocart a fait sur HCl dissous de diverses concentrations un grand nombre de mesures par la méthode d'ascension et par celle du pendule de translation et donne $-10^{6} \%_{\mathrm{HCl}}=22,0$ nombre probablement exact à $0, \breve{s}$ pour cent près. Cette valeur est appuyée par celle de Perrakis ( $\left.{ }^{\prime}\right), 22,1$ obtenue par un nombre moindre de mesures et par celle de Reicheneder (loc. cit.) égale à 21,9 .

Le coefficient d'aimantation de l'ion $\mathrm{Cl}-\mathrm{s}$ 'en déduit en retranchant le paramagnétisme apparent de $\mathrm{H}+$. On a donc :

$$
-10^{6} \chi_{\mathrm{Cl}^{-}}=23,1 \pm 0,6
$$

L'erreur propre de la mesure est probablement bien inférieure à celle qui peut provenir de la correction de l'effet de $\mathrm{H}+$ sur l'eau. J'ai indiqué l'approximation du résultat d'une manière qui paraît plausible en purtant après le nombre \pm la moitié de la correction. Je me propose de procéder de même pour les autres ions. Quand plusieurs correclions de déformation interviennent, je donnerai \pm leur demi-somme.

Le chlore n'ayant pas de répercussion sensible sur les molécules d'eau (Tableau I), le diamagnétisme de l'ion dissous ainsi trouvé est aussi celui de l'ion libre.

Il donne immédiatement le diamagnétisme des anions à partir des chlorures en solution. On a (Hocart) :

$$
-10^{6} \gamma_{\mathrm{NaCl}}=30,8 \quad-10^{6} \gamma_{\mathrm{KCl}}=39,6 \quad-10^{6} \gamma_{\mathrm{CaCl}}=55,6
$$

et

$$
-10^{6} \%_{\mathrm{Na}^{+}}=7,7 \pm 0,6 \quad-10^{6} \%_{\mathrm{K}^{+}}=16,5 \pm 0,6 \quad-10^{5} \%_{\mathrm{Ca}^{++}}=9,4 \pm 1,2
$$

Ce sont les diamagnétismes des anions dissous. Pour en déduire ceux des ions libres, il faut les corriger de la répercussion de l'anion sur l'eau au moyen de la formule (3) avec les valeurs du tableau I. On trouve :

$$
-10^{6} \Delta \%_{\mathrm{sa}^{+}}=0, \mathrm{~s} \quad-10^{6} \Delta \%_{\mathrm{K}^{+}}=0 \quad-10^{6} \Delta \%_{\mathrm{Ca}^{++}}=1,2
$$

d'où pour les ions libres :

$$
-10^{6} \%_{\mathrm{Na}^{+}}=8,2 \pm 0,9-10^{6} \%_{\mathrm{K}^{+}}=16,5 \pm 0,9 \quad-16^{6} \%_{\mathrm{Ca}^{++}}=10,6 \pm 1,8 .
$$

L'incertitude de $\%_{\mathrm{K}+}$ a été évaluée égale à celle de $\%_{\mathrm{Na}+}$ à cause du caractère approximalif possible de la donnée $\Delta R_{\mathrm{K}+}=0$.

Sels solides, Diamagnétisme de $\mathrm{Br}^{-}, \mathrm{I}^{-}$. - $\mathrm{KBr}$ et $\mathrm{KI}$ n'ont été étudiés par Hocart qu'à l'état cristallisé. Il a trouvé : $-10^{6} \%_{\mathrm{KB}_{2}}=19,1$ et $-10^{6} \%_{\mathrm{KI}}=63,8$. On peut,

(1) J. de Phys, s. VI, t. 8, p. 473. 
en s'appuyant eneore sur le rapport des sensibilités de la réfraction et du diamagnétisme à une mème déformation, calculer les corrections de dissolution par :

$$
\frac{\Delta \%_{\mathrm{KBr}}}{\chi_{\mathrm{Br}^{-}}}=\frac{1}{2} \frac{\Delta R_{\mathrm{KBr}}}{R_{\mathrm{Br}-}} \quad \frac{\Delta \%_{\mathrm{KI}}}{\chi_{\mathrm{I}^{-}}}=\frac{1}{2} \frac{\Delta R_{\mathrm{KI}}}{R_{\mathrm{I}^{-}}} .
$$

On a d'après Fajans et Joos :

$$
\begin{aligned}
\Delta R_{\mathrm{KBr}} & =0,92 & R_{\mathrm{Br}^{-}} & =12,67 \\
\Delta R_{\mathrm{KI}} & =1,72 & R_{\mathrm{I}^{-}} & =19,24
\end{aligned}
$$

et, en anticipant sur les résultats auxquels nous arriverons :

$$
-10^{6} \%_{\mathrm{Br}^{-}}=33, \mathrm{x}^{5} \quad-10^{6} \%_{\mathrm{I}^{-}}=49,2
$$

dont les valeurs approchées, sans correction, eussent été suffisantes pour l'usage que nous. en faisons ici ; d'où

$$
-10^{6} \Delta \gamma_{\mathrm{kBr}}=1,2 \mathrm{\partial} \quad \text { et } \quad-10^{6} \Delta \%_{\mathrm{KI}}=2,2
$$

ce qui donne pour les solutions :

$$
-10^{6} \chi_{\mathrm{KBr}}=50,4 \pm 0,6-10^{6} \chi_{\mathrm{KI}}=66,0 \pm 1,1
$$

et, en retranchant

$$
-10^{6} x_{K^{+}} \text {diss. }=13,3 \pm 0,6
$$

les diamagnétismes des ions dissous qui sont aussi ceux des ions libres :

$$
-10^{6} \gamma_{\mathrm{Br}^{-}}=33,9 \pm 1,2-10^{6} \gamma_{\mathrm{I}^{-}}=49,3 \pm 1,7 .
$$

Nos valeurs du diamagnétisme des ions halogènes cont voisines de celles que Reicheneder (loc. cit.) a obtenues par une étude soignée des trois hydracides en solution, mais par une méthode dont il estime lui-mème qu'elle ne se prète pas à une haute précision. Le tableau IV contient les valeurs des deux auteurs. Celles de Reicheneder sont, comme celles de Hocart, corrigées du paramagnétisme apparent de $\mathrm{H}+$.

TableaU IV.

$$
\begin{aligned}
& \text { Hocart. Reicheneder. } \\
& -10^{6} \%_{\mathrm{cl}-} \ldots \ldots \ldots \ldots \ldots .23,1 \pm 0,6 \quad \overline{23,0} \\
& -10^{6} \%_{\mathrm{Br}^{-}} \ldots \ldots \ldots \ldots \ldots .33,9 \pm 1,2 \\
& -10^{5} \% \text { I } \ldots \ldots \ldots \ldots \ldots .4 !, 5 \pm 1,7 \quad 51,3
\end{aligned}
$$

L'accord est d'autant plus remarquable que les valeurs déduites des mesures de Hocart ont été obtenues par une voie indirecte, en passant par les sels de $\mathbf{K}$ cristallisés.

Sels solides. Diamagnétisme de $\mathbf{M g}^{++}, \mathbf{C a}^{++}, \mathbf{S r}{ }^{++}$- Hocart a mesuré lecoefficient d'aimantation de $\mathrm{MgCl}^{2}, \mathrm{CaCl}^{2}, \mathrm{SrCl}^{2}(1)$ à létat cristallisé. On ne connaît pas-

(1) J'écarte la valeur de $\mathrm{BaCl}^{2},-10^{\circ} \%=72,2$ de Hocart que la divergence avec celle de Pascal, 78.0 rend suspecte. 
pour ces substances l'altération de la réfraction au passage de t'état solide à la zolation. Irais on peut évaluer l'altération du diamagnétisme de $\mathbf{M g C l}^{2}$ et de $\mathrm{SrCl}^{2}$ en s'appuyant sur la valeur connue pour $\mathrm{CaCl}^{2}$ par les mesures de Hocart :

$$
-10^{6}\left(\%_{\mathrm{CaCl}^{2} \text { diss. }}-\%_{\mathrm{CaCl}=}^{\mathrm{crist}}\right)=1,1 \% .
$$

A l'aide des valeurs du tableau $\mathrm{I}$ pour $\mathrm{Ca}^{+}+$, et $\mathrm{Cl}-$ on calcule par la formule (3)

$$
\begin{gathered}
-10^{6}\left(\%_{\mathrm{Ca}}+{ }_{\text {libre }}-\%_{\mathrm{Ca}^{+}+}{ }_{\text {diss }}\right)=1,15 \\
-10^{6}\left(\%_{\mathrm{Cl}-\text { libre }}-\%_{\mathrm{Cl}^{-} \text {diss. }}\right)=0
\end{gathered}
$$

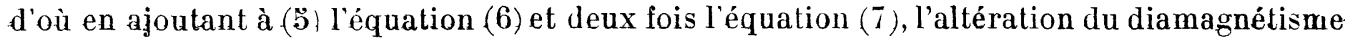
des ions libres dans la formation du cristal :

$$
-10^{6} \Delta \%=-10^{6}\left(\%_{\mathrm{Ca}}+{ }_{1 \mathrm{bbre}}+2 \%_{\mathrm{Cl}-\mathrm{libre}}-\%_{\mathrm{CaCl} \text { crist }}\right)=2,3 .
$$

L'allération dans le cristal porte sur l'anion, elle provient de l'attraction que le cation exerce sur lui et est proportionnelle au champ électrostatique de $\mathrm{Ca}++$ à l'endroit où se trouvent les $\mathrm{Cl}^{-}$voisins. Le même cation agit dans la solution sur les molécules neutres d'eau qu'il polarise. La compression qu'il exerce sur elles est proportionnelle au carré du champ. L'altération $\Delta \%$ par la réunion des ions libres dans le cristal est donc proportionnelle à la racine carrée de l'altération de la réfraction de l'eau par le cation. Mais cette dernière es't connue (tableau I). Il en résulte pour $\mathrm{Mg}$ et Sr les corrections données dans le tableau $\mathrm{V}$.

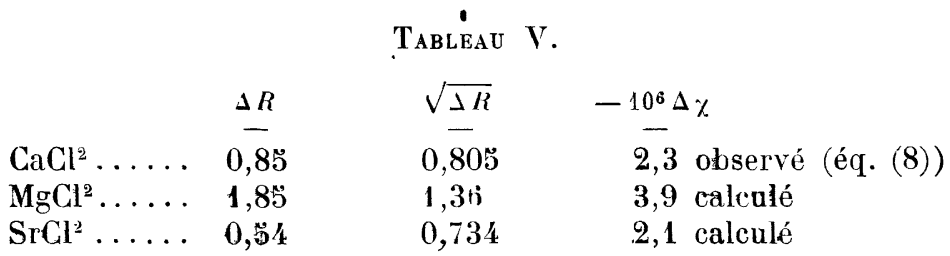

d'où, en partant des valeurs de Hocart :

$$
-10^{6} \%_{\mathrm{Mg}_{3} \mathrm{Cl}^{2} \text { erist. }}=47,45
$$$$
-10^{6} \%_{\mathrm{SrCl}^{2} \text { crist. }}=63,0
$$

pour les cations libres:

$$
-10^{6} \%_{\mathrm{Mg}^{+}}=5,15 \pm 2,6 \quad-10^{6} \psi_{\mathrm{sr}^{++}}=18,9 \pm 1,6 .
$$

Calcul des diamagnétismes des ions libres au moyen des diamagnétismes atomiques de Pascal (toc. cit.). - Les mesures de Pascal qui ont porté sur un très grand nombre de sels, n'ont pas été faites en recherchant une approximation qui ext permis de déceler les petites variations du diamagnétisme avec l'état phỹique. Mais nous avons déjà dit que dans les limites de leur précision, elles s'accordent avec celles de Hocart qui en reçoivent une confirmation utile. Nous allons déduire des nombres de Pascal, les diamagnétismes des ions, en nous appuyant sur notre valeur $-10^{6} \% \mathrm{cl}^{-}=23,1$. Mais comme les tableaux de Pascal contiennent, non les valeurs moléculaires, mais les valeurs atomiques, où le diamagnétisme de l'atome de chlore figure pour $-10^{6} \% \mathrm{cl}^{-}=20,1$, nous obtiendrons. le diamagnétisme des anions monovalents en corrigeant les diamagnétismes atomiques de $+3,0$, ceux des cations monovalents en corrigeant de $-3,0$, ceux des cations divalents en corrigeant de - ti,0, ete.

Nous avons fait la rẻduction aux ions libres comme ci-dessus, en admettant que les 
nombre de Pascal proviennent des chlorures solides et des halogénures de $\mathbf{K}$ (c'est le cas le plus fréquent), et nous avons trouvé :

TableaU VI.

\begin{tabular}{|c|c|c|c|c|}
\hline & $-10^{5} \%$ atom & CORRECTION DONIQTE & $\begin{array}{c}\text { CORRECTION DE Ŕ́DCCTION } \\
\text { ACX IONS LIBRES }\end{array}$ & $-11^{6} \%$ ion libre \\
\hline $\begin{array}{l}\mathrm{Na} \ldots \ldots \\
\mathbf{K} \ldots \ldots \ldots \\
\mathbf{R b} \ldots \ldots \ldots \\
\mathrm{Cs} \ldots \ldots \ldots\end{array}$ & $\begin{array}{r}9,2 \\
18,0 \\
27,2 \\
41,0\end{array}$ & $\begin{array}{c}-3,0 \\
» \\
» \\
»\end{array}$ & $\begin{aligned} &+ 1,4 \\
& 0,5 \\
& 0,1 \\
& 0\end{aligned}$ & $\begin{array}{r}7,6 \\
16,0 \\
24,3 \\
38,0\end{array}$ \\
\hline $\begin{array}{l}\mathrm{Cl} \ldots \ldots \ldots \\
\mathrm{Br} \ldots \ldots \\
\mathrm{I} \ldots \ldots \ldots\end{array}$ & $\begin{array}{l}20,1 \\
30,5 \\
44,6\end{array}$ & $\begin{array}{c}+: 2,0 \\
” \\
\prime\end{array}$ & $\begin{array}{r}\prime \prime \\
+\quad 1,2 \\
2,2\end{array}$ & $\begin{array}{l}23,1 \text { (admis) } \\
34,7 \\
49,8\end{array}$ \\
\hline $\begin{array}{l}\mathrm{Mg} \ldots \ldots \ldots \\
\mathrm{Ca} \ldots \ldots \ldots \\
\mathrm{Sr} . \ldots \ldots \ldots\end{array}$ & $\begin{array}{l}10,1 \\
15,9 \\
24,5\end{array}$ & $\begin{array}{c}-6,0 \\
" \\
"\end{array}$ & $\begin{aligned} &+ 3,9 \\
& 2,3 \\
& 2,1(?)\end{aligned}$ & $\begin{array}{r}8,0 \\
12,2 \\
20,6\end{array}$ \\
\hline
\end{tabular}

Comparaison avec la théorie. - Dans le tableau VII j’ai réuni les coefficients d'aimantation des ions libres calcu'ées par Pauling et par Stoner en s'appuyant sur leurs théories (loc. cit.) et ceux que j'ai déduits des observations de Hocart, de Reicheneder et de Pascal. J'y ai ajouté les valeurs concernant les gaz inertes de Hector et Wills.

TABIEAU VII.

\begin{tabular}{|c|c|c|c|c|c|c|}
\hline & \multicolumn{2}{|c|}{ THÉORTE } & \multicolumn{4}{|c|}{ EXPÉRIENCE } \\
\hline & PaUlixg & STONER & $\begin{array}{c}\text { HECTOR } \\
\text { ET WILLS }\end{array}$ & HOCART & REICHENEDER & PASCAL \\
\hline $\begin{array}{l}\mathrm{Cl}^{-} \ldots \ldots \ldots \\
\mathrm{Br}^{-} \ldots \ldots \ldots \ldots \\
\mathrm{I}^{-} \ldots \ldots \ldots \ldots\end{array}$ & $\begin{array}{l}29 \\
54 \\
80\end{array}$ & 40,39 & & $\begin{array}{l}23,1 \pm 1,6 \\
33,9 \pm 1,9 \\
49,3 \pm 1,7\end{array}$ & $\begin{array}{l}23,0 \\
33,6 \\
51,3\end{array}$ & $\begin{array}{l}33,1(\mathrm{adm} . \\
34,7 \\
49,8\end{array}$ \\
\hline $\begin{array}{l}\mathrm{He} \ldots \ldots \ldots \ldots \\
\mathrm{Ne} \ldots \ldots \ldots \ldots \\
\mathrm{A} \ldots \ldots \ldots \ldots\end{array}$ & $\begin{array}{c}1, \ddot{0} 4 \\
\check{3}, 7 \\
21, \breve{5}\end{array}$ & 1,90 & $\begin{array}{c}1,88 \\
6,7 \\
18,1\end{array}$ & & & \\
\hline $\begin{array}{l}\mathrm{Na}^{+} \ldots \ldots \ldots \\
\mathbf{K}^{+} \ldots \ldots \ldots \\
\mathbf{R}_{b^{+}} \ldots \ldots \ldots \\
\mathbf{C s}^{+} \ldots \ldots \ldots\end{array}$ & $\begin{array}{l}4,2 \\
16,7 \\
3 \ddot{3} \\
503\end{array}$ & $\begin{array}{r}5,47 \\
17,(11 \\
30,12\end{array}$ & & $\begin{array}{r}8,2 \pm 1,9 \\
16,3 \pm 0,9\end{array}$ & & $\begin{array}{r}7,6 \\
16,0 \\
21,3 \\
38,0\end{array}$ \\
\hline $\begin{array}{l}\mathrm{Mg}^{++} \ldots \ldots \ldots \\
\mathrm{Ca}^{++} \ldots \ldots \ldots \\
\mathrm{Sr}^{++} \ldots \ldots \ldots\end{array}$ & $\begin{array}{l}3,2 \\
13,3 \\
28\end{array}$ & & & $\begin{array}{r}5,15 \pm 2,6 \\
10,6 \pm 1,8 \\
18.9 \pm 1,6\end{array}$ & & $\begin{array}{r}8,0 \\
12,9 \\
20,6\end{array}$ \\
\hline
\end{tabular}

La valeur théorique de He déduite par Stoner des données électroniques sans qu'il soit fait aucun autre emprunt à l'expérience est remarquablement voisine de celle de Hector et Wills. Dans la théorie de Pauling, qui ne se suffit pas à elle-même, mais s'appuie sur des 
données réfractométriques, la vérification est aussi assez bonne pour les gaz inertes. Pour les ions des autres familles l'accord n'est plus satisfaisant. Dans les alcalins et alcalinoterreux, la théorie de Paulirg donne des valeurs trop faibles pour les ions de petits nombres atomiques et des valeurs trop fortes pour ceux de nombre atomique élevé. Cette conclusion subsiste mème si l'on écarte $\mathrm{Mg}++$ à cause de la correction de déformation un peu forte. La coïncidence se produit aux environs de $\mathbf{K}+$ qui pour cette raison paraît vérifier la théorie. L'excès de la théorie sur l'expérience aux nombres atomiques élevés s'aggrave encore dans la famille des halogènes ( $\left.{ }^{1}\right)$.

Dans la théorie de Stoner les écarts se produisent dans le mème sens : valeurs trop faibles pour les ions de petite masse et trop forte pour les ions de grande masse. Les exemples du chlore et du rubidium semblent indiquer que la divergence aux masses élevées est plus forte que dans la théorie de Pauling pour les halogènes et plus faible pour les alcalins.

On peut être tenté d'attribuer la divergence, comme le fait Stoner, à ce que la charge superficielle serait beaucoup plus diffuse dans les ions libres, auxquels se rapporte la théorie que dans les ions dissous. En effet, nous avons fait la correction de la déformation de l'eau par les ions, mais non celle de la déformation des ions par l'eau, estimée négligeable. Mais la régularité de la série des valeurs expérimentales de $\mathrm{Cl}-, \mathrm{A}, \mathrm{K}^{+}$et le fait qui parait certain de l'excès de la valeur expérimentale de $\mathrm{Na}+$ sur la valeur théorique semblent contredire à cette explication.

En résumé nous avons déduit les valeurs des diamagnétismes ioniques des mesures précises de Hocart avec l'aide des données réfractométriques de Fajans et Joos et en les appuyant des mesures de Reicheneder et de Pascal. L'excellente coïncidence des valeurs de l'hélium mise à part, il y a entre l'expérience et la théorie des divergences systématiques dépassant l'incertitude des mesures.

(1) La proportionnalité des coefficients d'aimantation des ions halogènes aux nombres atomiques que Joos (loc. cit.) a cru pouvoir déduire de données de précision insuffisante, ne se vérifie pas. Les quotients pour $\mathrm{Cl}^{-}, \mathrm{Br}^{-}, \mathrm{I}-$ sont $1,28,0,94 ; 0,92$. 